\title{
A narrative review of the clinical development of CDK4/6 inhibitor abemaciclib in breast cancer
}

\author{
Liu Yang", Yang Chen", Ning Wang, Weiwei Han \\ Eli Lilly and Company, Shanghai, China \\ Contributions: (I) Conception and design: L Yang, Y Chen, N Wang; (II) Administrative support: W Han; (III) Provision of study materials or \\ patients: L Yang, Y Chen, N Wang; (IV) Collection and assembly of data: L Yang, Y Chen; (V) Data analysis and interpretation: L Yang, Y Chen, W \\ Han; (VI) Manuscript writing: All authors; (VII) Final approval of manuscript: All authors. \\ "These authors contributed equally to this work. \\ Correspondence to: Weiwei Han. Eli Lilly and Company, 19F Tower 1 HKRI, Taikoo Hui, No. 288, Shi Men Yi Road, Shanghai 200041, China. \\ Email: Weiwei_han@lilly.com.
}

\begin{abstract}
Background and Objective: Advanced or metastatic breast cancer (MBC) is associated with poor prognosis and presents many challenges in medical management and treatment decisions. Anticancer drugs that act on cell cycle mechanisms have shown great potential in preclinical studies. In clinical trials, abemaciclib, a reversible ATP-competitive cyclin-dependent kinase 4/6 (CDK4/6) inhibitor developed by Eli Lilly and Company, combined with endocrine therapy (ET) were associated with superior outcomes compared with ET alone in patients with advanced or metastatic hormone receptor positive $\left(\mathrm{HR}^{+}\right) / \mathrm{human}$ epidermal growth factor receptor 2 negative (HER2 ${ }^{-}$) breast cancer, representing a new standard-of-care in this population. Abemaciclib has been approved by the U.S. Food and Drug Administration (FDA) for use in $\mathrm{HR}^{+} / \mathrm{HER} 2^{-}$MBC. In China, abemaciclib was also approved by the National Medical Products Administration (NMPA) based on findings from the MONARCH plus trial. Recently, abemaciclib have been approved as the first and only CDK4/6 inhibitor by FDA and NMPA for use in $\mathrm{HR}^{+} / \mathrm{HER} 2^{-}$, node-positive, early breast cancer $(\mathrm{EBC})$ at high risk of recurrence and Ki-67 score $\geq 20 \%$. Further trials of abemaciclib are ongoing. This is an overview of the clinical development of abemaciclib in breast cancer.
\end{abstract}

Methods: We reviewed English publications in PubMed related to CDK4/6 inhibitors from 2011 to 2021.

Key Content and Findings: In this review, we summarized the mechanism, results of preclinical and clinical studies of abemaciclib, describing current indications for treatment, ongoing clinical trials, safety and tolerability, and future perspectives.

Conclusions: Abemaciclib is a unique CDK4/6 inhibitor with distinctive characteristics and promising data, which bring benefit to $\mathrm{HR}^{+}, \mathrm{HER}^{-}$breast cancer patients.

Keywords: Cyclin-dependent kinase 4/6 inhibitor (CDK4/6 inhibitor); abemaciclib; hormone receptor positive/ human epidermal growth factor receptor 2 negative breast cancer (HR $/ \mathrm{HER}^{-}$breast cancer); metastatic breast cancer (MBC)

Received: 07 December 2021; Accepted: 21 January 2022.

doi: $10.21037 /$ tbcr-21-36

View this article at: https://dx.doi.org/10.21037/tbcr-21-36

\section{Introduction}

Female breast cancer has surpassed lung cancer as the most commonly diagnosed cancer, with an estimated 2.3 million new cases and 685,000 deaths worldwide (1). Estrogen receptor (ER) and/or progesterone receptor (PR) expression occurs in the majority $(60-75 \%)$ of breast cancers (2). Most of these tumors are initially dependent on activation of ERs by the steroid hormone estrogen.

Endocrine therapy (ET), including selective ER 
Table 1 The search strategy summary

\begin{tabular}{ll}
\hline Items & Specification \\
\hline Date of search (specified to date, month and year) & Nov 1, 2021 \\
Databases and other sources searched & PubMed, FDA and NMPA website \\
$\begin{array}{l}\text { Search terms used (including MeSH and free text search terms and filters). Note: please use an } \\
\text { independent supplement table to present detailed search strategy of one database as an example }\end{array}$ & CDK4/6 inhibitor, abemaciclib, \\
Himeframe & $2011-2021$ \\
Inclusion and exclusion criteria (study type, language restrictions etc.) & None \\
Selection process (who conducted the selection, whether it was conducted independently, how & None \\
consensus was obtained, etc.) & None \\
Any additional considerations, if applicable &
\end{tabular}

FDA, Food and Drug Administration; NMPA, National Medical Products Administration; CDK4/6, cyclin dependent kinase 4/6; HR ${ }^{+}$ hormone receptor positive; HER2 ${ }^{-}$, human epidermal growth factor receptor 2 negative; MBC, metastatic breast cancer.

modulators (SERMs), aromatase inhibitors (AIs), and selective ER down-regulators (SERDs), has become the standard-of-care for patients with early or advanced hormone receptor positive $\left(\mathrm{HR}^{+}\right) /$human epidermal growth factor receptor 2 negative (HER2-) breast cancer, and has significantly improved survival in this population. However, almost all patients with advanced or metastatic disease will develop disease progression due to primary and secondary endocrine resistance (3). Although the mechanisms of endocrine resistance in patients with $\mathrm{HR}^{+}$breast cancer are complex and remain to be fully understood, substantial insights have been gained in recent years. Through the study of endocrine drug resistance mechanisms and use of corresponding targeted drugs combined with various ETs, significant clinical benefits have been achieved for patients with HR+ advanced breast cancer. Currently approved targeted drugs in this setting include phosphoinositide-3kinase (PI3K)-mammalian target of rapamycin (mTOR) inhibitor (such as everolimus) (4), cyclin-dependent kinase 4/6 (CDK4/6) inhibitors (such as abemaciclib) (5-7), subtype-selective histone deacetylase (HDAC) inhibitor (such as chidamide) (8), and alpha-specific PI3K inhibitor (such as alpelisib) (9).

CDK4 and CDK6, which are types of serine-threonine kinases, are key regulators of the cell cycle that mediate the transition from G1 phase (prophase of DNA synthesis) to $\mathrm{S}$ phase (DNA synthesis) by binding to cyclin D. Dysregulation of the cyclin D-CDK4/6-inhibitor of CDK4 (INK4)-retinoblastoma tumor suppressor protein $(\mathrm{Rb})$ pathway is present in many tumors, including breast cancer, and the changes in this pathway accelerate the G1 phase process and drive tumor cell proliferation. Therefore, inhibition of this pathway has become a key area of interest in the research and development of new anticancer drugs, and CDK4/6 is emerging as an important anti-tumor target $(10,11)$. In this work, we reviewed English publications related to CDK4/6 inhibitors in recent 10 years (Table 1) and focus on the results of preclinical and clinical studies of abemaciclib, a CDK4/6 inhibitor, describing the current indications for treatment, ongoing clinical trials, safety and tolerability, and future perspectives.

We present the following article in accordance with the Narrative Review reporting checklist (available at https:// tbcr.amegroups.com/article/view/10.21037/tbcr-21-36/rc).

\section{Methods}

See Table 1.

\section{CDK4/6 and CDK4/6 inhibitors}

\section{Cell cycle regulation and CDK4/6}

The mammalian cell cycle is a highly organized and regulated process that ensures duplication of genetic material and cell division. Cyclins and CDKs are two types of proteins involved in cell cycle regulation. A subset of cyclin-CDK complexes is directly involved in cell cycle progression, including ten cyclins that belong to four different classes (the A-, B-, D- and E-type cyclins), three interphase CDKs (CDK2, CDK4 and CDK6), and a mitotic CDK (CDK1) (10). The CDK4/cyclin D and CDK6/ cyclin D complexes are key regulators of the G1 phase at 


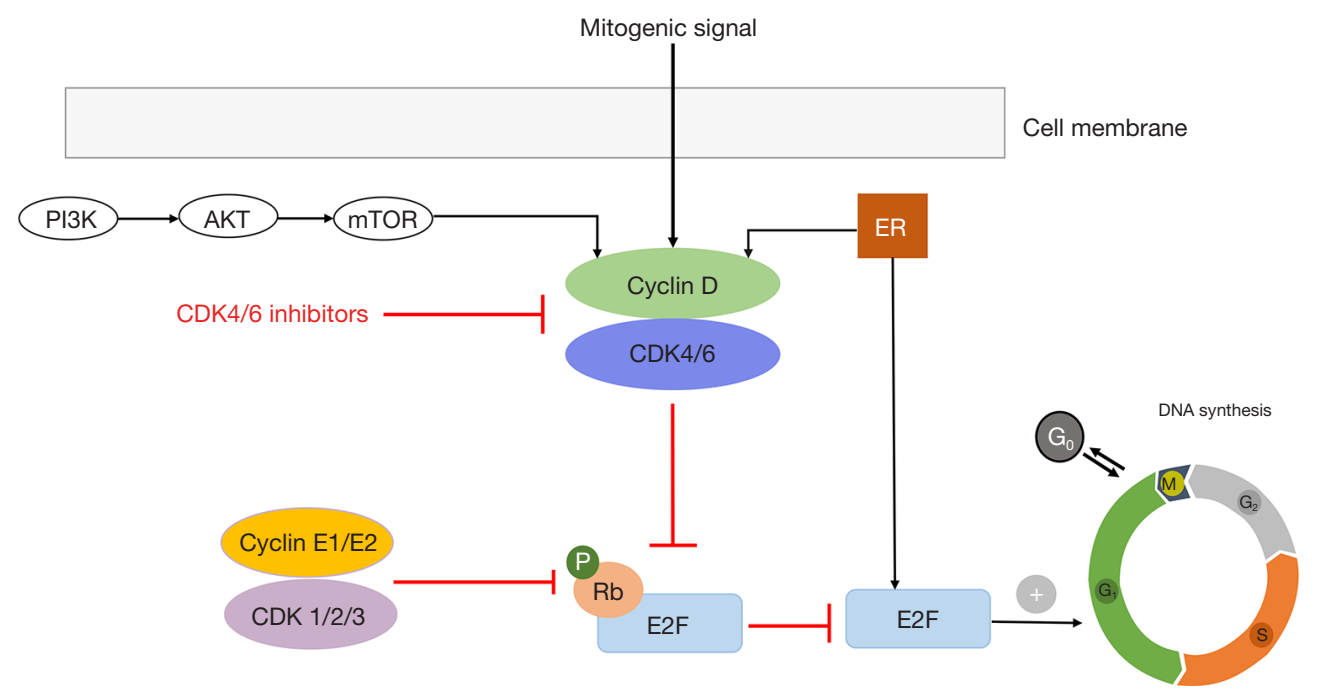

Figure 1 Mechanism of action of CDK4/6 inhibitors. The CDK4/6-cyclin D complex induces phosphorylation of the Rb. Free transcription factor E2F stimulates cell transition from the G1 to the S phase and cell division. CDK4/6, cyclin dependent kinase 4/6; Rb, retinoblastoma tumor suppressor protein; E2F, transcription factor family; ER, estrogen receptor; mTOR, mammalian target of rapamycin; AKT, protein kinase B; PI3K, phosphoinositide 3-kinase.

the molecular level, driving cell proliferation by several mechanisms. Most importantly, estrogen stimulates cyclin $\mathrm{D}$ expression during cell cycle progression, promoting CDK 4 and CDK6 activation $(12,13)$. The Rb was phosphorylated by cyclin D-CDK4/6 and the transcription factor was released to promote cell cycle progression to $\mathrm{S}$ phase, ultimately leading to cell proliferation. Amplification or overexpression of CDK4 and CDK6 have been observed in several malignancies $(10,11)$, including breast cancer.

\section{CDK inbibitors}

CDK4 and CDK6 have long been considered potential therapeutic targets. However, progress towards development of inhibitors for these kinases has been limited by issues with potency, selectivity, and poor pharmacokinetic (PK) and pharmacodynamic properties. The first generation of CDK4 and CDK6 inhibitors are primarily pan-CDK inhibitors (such as flavopiridol). The clinical results are disappointing, especially an unfavorable safety profile $(14,15)$. The second generation of CDK inhibitors (such as dinaciclib) had better selectivity, but the usage was restrained by severe toxicities and limited efficacy (16). The next generation of CDK inhibitors had further improved selectivity (Figure 1), specifically targeting CDK4 and CDK6 (17-19), and were associated with better overall efficacy and safety in patients. At present, there are three selective CDK4/6 inhibitors approved globally for the treatment of advanced or metastatic $\mathrm{HR}^{+} / \mathrm{HER} 2^{-}$breast cancer, namely palbociclib (PD 0332991; Pfizer, Inc.), abemaciclib (LY2835219; Eli Lilly and Company), and ribociclib (LEE011; Novartis International AG). Moreover, abemaciclib is the first and only Food and Drug Administration (FDA)-approved CDK4/6 inhibitor for the adjuvant treatment of $\mathrm{HR}^{+}$, HER2-, node-positive, Ki-67 score $\geq 20 \%$ early breast cancer (EBC) at high risk of recurrence. Palbociclib, abemaciclib and dalpiciclib (SHR6390; Jiangsu Hengrui Medicine Co., Ltd., Lianyungang, China). are currently approved for the treatment of advanced or metastatic $\mathrm{HR}^{+}$/ HER2 ${ }^{-}$breast cancer in China, and abemaciclib is the only CDK4/6 inhibitor approved for use in both metastatic breast cancer (MBC) and EBC in China.

\section{Preclinical discovery and development of abemaciclib in breast cancer}

\section{Discovery and preclinical development}

LY2835219 (abemaciclib) was discovered by scientists at Eli Lilly and Company Research Laboratories. Abemaciclib (Figure 2) binds to inactive kinase conformation of CDK4/6 and establishes a reversible interaction. Two hydrogen bonds are established in the three CDK4/6 inhibitors 


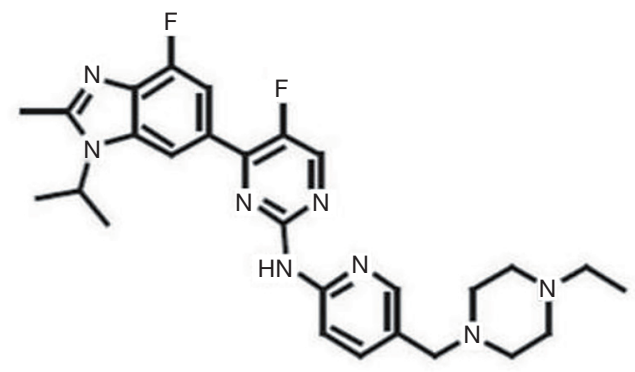

Figure 2 Chemical structure of LY2835219/abemaciclib.

with residues in the hinge region of CDK4/6 ATP pocket. One hydrogen bond is between the pyridine $\mathrm{N}$ atom and ${ }^{H i s 100} \mathrm{NH}$, and another one is between the exocyclic $\mathrm{NH}$ of the side chain and ${ }^{\text {Vallo1 }} \mathrm{CO}$. The amino side chains of these inhibitors are observed to occupy different positions. Compared with abemaciclib, ribociclib and palbociclib bind differently to CDK4/6 and have larger substituents, while abemaciclib has only a fluorine atom. Therefore, abemaciclib is more deeply buried into the ATP cleft than ribociclib and palbociclib (20).

Abemaciclib was selected for its biological activity, as well as the highly selective inhibition of CDK4/ cyclin D1 $\left(\mathrm{Ki}^{\mathrm{ATP}}=0.6 \pm 0.3 \mathrm{nmol} / \mathrm{L}\right)$ and CDK6/cyclin D3 $\left(\mathrm{Ki}^{\mathrm{ATP}}=8.2 \pm 1.1 \mathrm{nmol} / \mathrm{L}\right)$ complexes $(21)$. There was no activity against other important cell-cycle-related kinases. In vitro, ER positive $\left(\mathrm{ER}^{+}\right)$breast cancer cells treated with abemaciclib alone led to a decrease in pRb, G1 arrest, and a decrease in cell proliferation, with durable inhibition even after drug removal. Abemaciclib also induced a senescence response, and with continuous exposure, induced apoptosis and altered breast cancer cell metabolism (21). In vivo, abemaciclib inhibited tumor growth in multiple human cancer xenograft models, including those derived from nonsmall cell lung cancer (NSCLC), melanoma, glioblastoma, mantle cell lymphoma and $\mathrm{ER}^{+}$breast cancer (17).

CDK4/cyclin D1 is associated with cell proliferation and is critical for maintaining the growth of breast cancer $(21,22)$, while CDK6/cyclin D3 is associated with the hematopoietic system and is important for normal thymus development and the maturation of bone marrow hematopoietic stem cells (22). Abemaciclib acts as a competitive inhibitor of the ATP-binding domain of CDK4 and CDK6, with 14 times greater potency against CDK4 compared with CDK6 (21). This selectivity for CDK4 suggests that abemaciclib may strongly inhibit the proliferation of breast cancer cells. Moreover, the relatively lower inhibitory effect of abemaciclib on CDK6 may reduce the likelihood of bone marrow toxicity. Therefore, these properties indicate that abemaciclib may represent the first selective CDK4/6 inhibitor that permits a continuous dosing schedule to produce durable target inhibition. Shortterm inhibition of CDK4/6 can lead to cell cycle recovery and induce the rebound of DNA synthesis. However, continuous inhibition of CDK4/6 will lead to continuous cell cycle arrest and apoptosis (21). Abemaciclib also has the ability to cross the blood-brain barrier, with similar drug concentrations in the cerebrospinal fluid and plasma.

In addition to cell-cycle dependent activity, CDK4/6 inhibitors have been shown to increase the frequency of CD8-positive T cell memory precursors and downregulate the expression of MYC target genes, suggesting a potential for augmentation of long-term protective immunity in patients with cancer (23). Furthermore, abemaciclib has been shown to promote antitumor immunity by simultaneously potentiating tumor antigen presentation, as well as selectively suppressing proliferation of regulatory $\mathrm{T}$ (Treg) cells (24).

\section{Phase I study}

Based on promising preclinical findings, a multicenter study was conducted including phase I dose escalation and tumor-specific cohorts for heavily pre-treated breast cancer, NSCLC, glioblastoma, melanoma and colorectal cancer (25). In this study, the maximum tolerated dose of abemaciclib was $200 \mathrm{mg}$ every 12 hours. The dose-limiting toxicity was grade 3 fatigue. Single-agent abemaciclib was well tolerated, supporting its potential as the first selective CDK4/6 inhibitor with a safety profile allowing continuous dosing to sustained CDK4/6 inhibiting in a variety of cancer types. The objective response rate (ORR) was $31 \%$ and the clinical benefit rate (CBR) was as high as $61.1 \%$ in patients with $\mathrm{HR}^{+}$breast cancer (25).

\section{The MONARCH series}

\section{MONARCH 1}

The phase I results prompted a phase II single-arm study (MONARCH 1) of abemaciclib monotherapy administered every 12 hours orally at a dose of $200 \mathrm{mg}$ in patients with refractory $\mathrm{HR}^{+} / \mathrm{HER} 2^{-} \mathrm{MBC}$ (26). The ORR was $19.7 \%$ [95\% confidence interval (CI): 13.3-27.5\%; null hypothesis of $15 \%$ not excluded], the median progression-free survival 
(PFS) was 6 months, and the median overall survival (OS) was 17.7 months. The most common grade 3 or 4 adverse events (AEs) were white blood cell decreased (27.7\%), neutrophil count decreased (26.9\%), diarrhea (19.7\%), and fatigue $(12.9 \%)$. Most patients with diarrhea did not require adjustment of the treatment dose. The majority of neutropenia events were grade 1 or 2 and not associated with fever and/or infection.

The results from MONARCH 1 prompted FDA to grant breakthrough therapy status to abemaciclib monotherapy in October 2015 for patients with refractory $\mathrm{HR}^{+}$advanced or MBC.

\section{MONARCH 2}

MONARCH 2 is a phase III study of abemaciclib $150 \mathrm{mg}$ twice daily or placebo combined with fulvestrant $500 \mathrm{mg}$ in women with $\mathrm{HR}^{+} / \mathrm{HER} 2^{-}$advanced breast cancer and disease progression while receiving ET (27). Median PFS, the primary endpoint, was $16.4 \mathrm{vs} .9 .3$ months in the abemaciclib arm and placebo arm, respectively [hazard ratio (HR), 0.553; 95\% CI: 0.449-0.681; $\mathrm{P}<0.001$ ]. The ORR was $48.1 \%$ (95\% CI: $42.6-53.6 \%$ ) vs. $21.3 \%$ (95\% CI: $15.1-27.6 \%$ ) in the abemaciclib arm and placebo arm in patients with measurable disease. The most common AEs in the abemaciclib $v s$. placebo arms were diarrhea $(86.4 \%$ vs. $24.7 \%)$, neutropenia (46.0\% vs. $4.0 \%$ ), nausea ( $45.1 \%$ vs. $22.9 \%$ ), and fatigue (39.9\% vs. $26.9 \%)$. The most frequently reported grade 3 or 4 AEs in the abemaciclib $v s$. placebo arms were neutropenia ( $26.5 \%$ vs. $1.7 \%)$, diarrhea (13.4\% vs. $0.4 \%$ ) and leukopenia (8.8\% vs. $0 \%)$.

Given the promising outcomes of MONARCH 1 and MONARCH 2, in September 2017, abemaciclib received FDA approval as monotherapy for $\mathrm{HR}^{+} / \mathrm{HER}^{-}$advanced or MBC with disease progression following ET and prior chemotherapy in the metastatic setting. Abemaciclib also received FDA approval for use in combination with fulvestrant in $\mathrm{HR}^{+} / \mathrm{HER} 2^{-}$advanced or $\mathrm{MBC}$ with disease progression after ET.

\section{MONARCH 3}

MONARCH 3 is a phase III study of abemaciclib in postmenopausal patients with $\mathrm{HR}^{+} / \mathrm{HER} 2^{-}$advanced breast cancer and no prior systemic treatment (6). Patients received abemaciclib (150 $\mathrm{mg}$ twice daily continuously) or placebo combined with an $\mathrm{AI}$ (anastrozole $1 \mathrm{mg}$ or letrozole $2.5 \mathrm{mg}$ daily). Unlike MONARCH 1 and MONARCH
2, which did not define patients' menopausal status, MONARCH 3 only included postmenopausal patients. The primary endpoint was investigator-assessed PFS. In the preplanned final analysis, median PFS was significantly longer in the abemaciclib arm compared with the placebo arm (28.18 vs. 14.76 months; HR, 0.540; 95\% CI: 0.4180.698; $\mathrm{P}=0.000002$ ) (28). For patients with measurable disease, the ORR was $61.0 \%$ and $45.5 \%$ in the abemaciclib arm and placebo arm, respectively $(\mathrm{P}=0.003)$. Diarrhea was the most frequent $\mathrm{AE}(82.3 \%)$ in the abemaciclib arm but was mainly grade $1(42.5 \%)$. The most frequently reported grade 3 or 4 AEs in the abemaciclib and placebo arms were neutropenia (23.9\% vs. $1.2 \%)$, diarrhea (9.5\% vs. $1.2 \%)$ and leukopenia (8.6\% vs. $0.6 \%)(28)$.

Based on MONARCH 3 results, in February 2018, abemaciclib received FDA approval for use in combination with an $\mathrm{AI}$ as initial endocrine-based treatment in postmenopausal women with $\mathrm{HR}^{+} / \mathrm{HER} 2^{-}$advanced or MBC.

\section{MONARCH plus}

\section{Rationale}

Evidence from multiple global phase III studies suggests that abemaciclib in combination with standard ET has significant antitumor activity and good tolerability in $\mathrm{HR}^{+} /$ HER2 ${ }^{-}$advanced breast cancer patients $(6,27)$. However, there is a lack of phase III clinical studies to verify the efficacy and safety profile of CDK4/6 inhibitors in Chinese patients. In addition, few of the available studies included patients in developing countries with large populations, such as Brazil, India and South Africa. Moreover, in these populations, the lack of data is compounded by limited access to CDK4/6 inhibitors. Therefore, clinical studies evaluating the safety and efficacy of abemaciclib in combination with ET for patients in developing countries are urgently needed.

An open-label phase I trial was conducted in Chinese patients with advanced malignancies (29) to evaluate the safety, tolerability, and PK characteristics of abemaciclib. A total of 26 patients were randomly assigned $1: 1$ to abemaciclib $150 \mathrm{mg}(\mathrm{n}=12)$ or $200 \mathrm{mg}(\mathrm{n}=13)$. Safety was the primary endpoint. Of the 25 patients included in the safety analysis who received at least one dose of abemaciclib, all reported $\geq 1$ treatment emergent $\mathrm{AE}$ (TEAE). The severity of most TEAEs were grade 1 or 2 . The most common grade $\geq 3$ TEAEs were neutropenia 
(32\%) and thrombocytopenia (24\%). There were 4 patients (16\%) discontinued treatment due to AEs. A single dose of abemaciclib showed slow absorption and clearance, with the maximum concentrations reached after about $6 \mathrm{~h}$ and an elimination half-life of about $24 \mathrm{~h}$. These results suggested that abemaciclib is well tolerated in Chinese patients, with similar safety and PK profiles to those previously reported in non-Chinese patients, thus supporting the further development of abemaciclib in the Chinese population.

\section{Study design features}

The MONARCH plus trial was designed by Lilly China Drug Development and Medical Affairs Center in conjunction with two eminent Chinese breast cancer scholars, Prof. Zefei Jiang and Prof. Xichun Hu. Through an innovative research and development concept and a novel trial design, this critical phase III trial with two treatment arms formed the basis of the approval of abemaciclib in the Chinese mainland, not only as initial treatment for advanced breast cancer patients in combination with AIs, but also in combination with fulvestrant for patients with breast cancer who had progressed on prior ET.

The inclusion criteria for cohort $\mathrm{A}$ and cohort $\mathrm{B}$ of MONARCH plus were similar to those for MONARCH 3 and MONARCH 2, respectively, with some adjustments to help answer unresolved questions in the previous two trials. MONARCH 3 did not determine whether patients who relapsed within 12 months of completing adjuvant ET could benefit from abemaciclib plus a non-steroidal AI (NSAI). This is particularly relevant in China, where the age of breast cancer onset is about 5 to 10 years earlier than that in western countries (30), and a large proportion of patients who relapse on adjuvant anti-estrogen therapy are still eligible to receive NSAIs. In the MONARCH plus study, cohort A was defined to include all patients eligible for treatment with NSAIs, namely those who had never received or were still considered sensitive to treatment with NSAIs. Cohort A included patients who relapsed within 1 year after completion of non-NSAI adjuvant ET, while cohort B included patients who relapsed within 1 year after completion of adjuvant ET with a NSAI.

\section{Pivotal data}

The MONARCH plus study (31) evaluated the efficacy and safety of abemaciclib combined with a NSAI or fulvestrant in postmenopausal patients with $\mathrm{HR}^{+} / \mathrm{HER} 2^{-}$advanced breast cancer.

Patients were enrolled from 45 centers in China, Brazil, India and South Africa from December 2016 to August 2018 , with more than $80 \%$ of the study population from China. In cohort A, 306 patients were 2:1 randomly assigned to abemaciclib plus NSAI $(n=207)$ or placebo plus NSAI ( $n=99)$. PFS was significantly improved in the abemaciclib arm compared with the placebo arm (median, not reached vs. 14.7 months; HR, 0.499; 95\% CI: 0.346$0.719 ; \mathrm{P}=0.0001)$. For patients with measurable disease, the ORR was $65.9 \%$ and $36.1 \%$ in the abemaciclib and placebo arm respectively $(\mathrm{P}<0.0001)$. In cohort $\mathrm{B}, 157$ patients were randomized 2:1 to abemaciclib plus fulvestrant $(\mathrm{n}=104)$ or placebo plus fulvestrant $(\mathrm{n}=53)$. The abemaciclib arm had a significantly improved median PFS compared with the placebo arm (median, 11.5 vs. 5.6 months; HR, 0.376; 95\% CI: $0.240-0.588$; $\mathrm{P}<0.0001)$. For patients with measurable disease, the ORR was $50.0 \%$ and $10.5 \%$ in the abemaciclib and placebo arm respectively $(\mathrm{P}<0.0001)$. The most common grade $\geq 3$ AEs in the abemaciclib arms were neutropenia, leukopenia, and anemia (both cohorts), and lymphocytopenia (cohort B).

Overall, abemaciclib in combination with a NSAI or fulvestrant demonstrated clinically and statistically significant improvements in PFS and ORR in $\mathrm{HR}^{+} / \mathrm{HER} 2^{-}$ advanced breast cancer patients, most of whom were from China. The efficacy results of MONARCH plus were consistent with those of the global MONARCH 2 and 3 trials and were also generally consistent across patient subgroups. Moreover, abemaciclib was well tolerated in combination with a NSAI or fulvestrant in patients from China, Brazil, India and South Africa, and no new safety signals were observed.

In December 2020, the National Medical Products Administration (NMPA) of China officially approved abemaciclib for breast cancer treatment, based on the interim results of MONARCH plus. This study is the first international phase III study to demonstrate the efficacy and safety of a CDK4/6 inhibitor in Chinese patients with $\mathrm{HR}^{+}$/ HER2 ${ }^{-}$advanced breast cancer. Based on the indications approved in China, abemaciclib in combination with an AI or fulvestrant was given a grade 1 recommendation in the Chinese Society of Clinical Oncology (CSCO) Breast Cancer Guidelines 2021 for $\mathrm{HR}^{+} / \mathrm{HER}^{-}{ }^{-}$advanced breast cancer (32).

\section{Other critical trials in the MONARCH series}

Given the success of CDK4/6 inhibition combined with ET 
in the treatment of $\mathrm{HR}^{+} / \mathrm{HER} 2^{-}$breast cancer, this strategy is also under consideration for $\mathrm{HR}^{+} / \mathrm{HER} 2^{+}$advanced breast cancer, with evidence shown that HER2 ${ }^{+}$breast tumors in resistance to trastuzumab may be partly caused by cyclin D1 overexpression (33). The phase II monarcHER study (34) evaluated abemaciclib + trastuzumab + fulvestrant $v s$. trastuzumab + chemotherapy in $\mathrm{HR}^{+} / \mathrm{HER} 2^{+}$advanced breast cancer patients who had received at least 2 lines of anti-HER2 therapy. The median PFS and ORR were improved with triple therapy, confirming that this regimen can provide clinical benefit in heavily pretreated patients with $\mathrm{HR}^{+} / \mathrm{HER}^{+}$breast cancer. These findings suggest that a chemotherapy-free regimen may be an alternative treatment option for $\mathrm{HR}^{+} / \mathrm{HER} 2^{+}$advanced breast cancer patients, although further studies are needed.

In addition to the research in advanced breast cancer, CDK4/6 inhibitors are also being explored as adjuvant therapy for patients with EBC. Interim results from the phase III monarchE study (35) suggested that abemaciclib in combination with standard adjuvant ET has efficacy in patients with high risk $\mathrm{HR}^{+} / \mathrm{HER} 2^{-} \mathrm{EBC}$, representing the only positive clinical evidence for CDK4/6 inhibitors in this setting. Based on monarchE results, abemaciclib became the first FDA-approved CDK4/6 inhibitor in EBC (36). In addition, the phase III eMonarcHER study (37) is ongoing in $\mathrm{HR}^{+} / \mathrm{HER} 2^{+}$EBC patients, including significant proportion of Chinese patients, and we look forward to the disclosure of results in the future.

\section{Safety \& tolerability of abemaciclib}

In general, abemaciclib associated AEs in the MONARCH trials were predictable, manageable, and usually reversible by dose adjustment.

The most common AEs with abemaciclib were gastrointestinal and hematologic events and fatigue. Abemaciclib had a lower incidence of neutropenia compared with palbociclib and ribociclib. This difference may be related to higher and more selective activity of abemaciclib against CDK4 vs. CDK6. Most cases of neutropenia with abemaciclib were observed early in the treatment course, reversed quickly and were managed with dose modifications. Diarrhea, the most common AE of abemaciclib, usually occurred early in the treatment course and was quickly resolved with conventional antidiarrhea medications and dose adjustment. In MONARCH 1, 2 and 3, 82.3-90.2\% patients developed diarrhea after receiving abemaciclib; most events were grade 1 or $2,9.5-19.7 \%$ of patients had grade 3 diarrhea, and grade 4 diarrhea was not reported (38).

Hepatotoxicity is a known AE of special interest for abemaciclib. In the abemaciclib arms of MONARCH 2 and 3 , the incidences of increased alanine transaminase (ALT) and aspartate transaminase (AST) at any grade were $13.4-17.4 \%$ and $12.2-16.8 \%$, respectively. In both studies, the abemaciclib arms had low rates of grade $\geq 3$ increased ALT and AST, and of increased ALT and AST $\geq 3$ times the upper limit of normal (ULN) with total bilirubin $\geq 2$ times the ULN (MONARCH 2, $0.2 \%$ and $0.5 \%$; MONARCH $3,0.3 \%$ and $0 \%$, respectively). In MONARCH 2 and 3, hepatotoxicity was reversible, with a short duration of grade $\geq 3$ ALT and AST elevation and a median time from onset to resolution (for all patients regardless of dose adjustment) of about 2 weeks. Dose reduction or discontinuation due to increased ALT or AST was uncommon $(<1 \%)$.

Abemaciclib has been shown to increase serum creatinine. This is caused by inhibition of renal tubular secretion transporters, and not affecting glomerular function. In clinical studies, increases in serum creatinine (mean increase, $0.2-0.3 \mathrm{mg} / \mathrm{dL}$ ) was shown within the first 28-day cycle of abemaciclib dosing. The serum creatinine was remained elevated but stable during treatment, and were reversible upon treatment discontinuation. In addition, serum creatinine increases were not concomitant with changes in alternative renal function markers that are not based on creatinine, such as cystatin C, blood urea nitrogen, or calculated glomerular filtration rate (39).

Venous thromboembolism events (VTEs) were infrequent and were reported more often in abemaciclib arm than placebo arm in MONARCH 2 (4.8\% vs. $0.9 \%)$ and MONARCH 3 (6.1\% vs. 0.6\%) (38). However, VTEs are not specific to abemaciclib and have been shown with other CDK4/6 inhibitors. A recently reported retrospective analysis in 424 patients treated with CDK4/6 inhibitors (91.8\% of patients received palbociclib) showed a 1 -year cumulative incidence of VTEs of $6.3 \%$ (40). Additional safety concerns were CDK4/6 inhibitor-induced interstitial lung disease (ILD) and/or pneumonitis. In clinical trials of CDK4/6 inhibitors, $1-3 \%$ of patients developed ILD and/ or pneumonitis of any grade, which was a fatal complication in $<1 \%$ of patients $(41)$.

\section{Future perspective}

The discovery and application of CDK4/6 inhibitors including abemaciclib have changed the treatment paradigm for $\mathrm{HR}^{+} / \mathrm{HER} 2^{-} \mathrm{MBC}$ and even high risk EBC. With the 
increasing clinical use of abemaciclib and other CDK4/6 inhibitors, there are several questions that need to be answered for patients with $\mathrm{HR}^{+} / \mathrm{HER} 2^{-}$metastatic and high risk EBC.

Firstly, abemaciclib does not provide benefit in all patients with $\mathrm{HR}^{+} / \mathrm{HER} 2^{-}$advanced breast cancer. Great efforts have been made to identify the mechanism of resistance to CDK4/6 inhibitors $(42,43)$, however, no clear mechanism of resistance is sufficiently validated. The identification of specific biomarkers could help to identify patients who are more likely to respond to treatment of CDK4/6 inhibitors. Several attempts have been made to find the biomarkers for response prediction through tissue biopsy or liquid biopsy. But for now, no clear biomarker is identified (44).

Secondly, it is unknown whether abemaciclib's biological ability to cross the blood-brain barrier could translate into a future treatment option for patients with breast cancer and brain metastases.

Thirdly, with the increasing use of CDK4/6 inhibitors, suitable therapies for patients with disease progression on CDK4/6 inhibitors need to be identified. It will be important to determine whether subsequent therapy could involve another CDK4/6 inhibitor. In addition, as several new drugs are being applied in the clinic, appropriate drug selection for combination with CDK4/6 inhibitors is a direction for future research and development.

In conclusion, CDK4/6 inhibitors including abemaciclib, are a useful addition to the treatment armamentarium for $\mathrm{HR}^{+} / \mathrm{HER} 2^{-}$advanced breast cancer.

\section{Acknowledgments}

We thank Sharon Gladwin, $\mathrm{PhD}$ (Rude Health Consulting) for the editorial support for this review article.

Funding: This work was supported by Eli Lilly and Company.

\section{Footnote}

Reporting Checklist: The authors have completed the Narrative Review reporting checklist (available at https:// tbcr.amegroups.com/article/view/10.21037/tbcr-21-36/rc).

Peer Review File: Available at https://tbcr.amegroups.com/ article/view/10.21037/tbcr-21-36/prf

Conflicts of Interest: All authors have completed the
ICMJE uniform disclosure form (available at https://tbcr. amegroups.com/article/view/10.21037/tbcr-21-36/coif). NW is a former employee of Eli Lilly and Company. LY, YC, and WH are current employees of Eli Lilly and Company.

Ethical Statement: The authors are accountable for all aspects of the work in ensuring that questions related to the accuracy or integrity of any part of the work are appropriately investigated and resolved.

Open Access Statement: This is an Open Access article distributed in accordance with the Creative Commons Attribution-NonCommercial-NoDerivs 4.0 International License (CC BY-NC-ND 4.0), which permits the noncommercial replication and distribution of the article with the strict proviso that no changes or edits are made and the original work is properly cited (including links to both the formal publication through the relevant DOI and the license). See: https://creativecommons.org/licenses/by-nc-nd/4.0/.

\section{References}

1. Sung H, Ferlay J, Siegel RL, et al. Global Cancer Statistics 2020: GLOBOCAN Estimates of Incidence and Mortality Worldwide for 36 Cancers in 185 Countries. CA Cancer J Clin 2021;71:209-49.

2. Aggelis V, Johnston SRD. Advances in Endocrine-Based Therapies for Estrogen Receptor-Positive Metastatic Breast Cancer. Drugs 2019;79:1849-66.

3. Ning Z. Why ACE-overview of the development of the subtype-selective histone deacetylase inhibitor chidamide in hormone receptor positive advanced breast cancer. Transl Breast Cancer Res 2020;1:5.

4. Baselga J, Campone M, Piccart M, et al. Everolimus in postmenopausal hormone-receptor-positive advanced breast cancer. N Engl J Med 2012;366:520-9.

5. Turner NC, Ro J, André F, et al. Palbociclib in HormoneReceptor-Positive Advanced Breast Cancer. N Engl J Med 2015;373:209-19.

6. Goetz MP, Toi M, Campone M, et al. MONARCH 3: Abemaciclib As Initial Therapy for Advanced Breast Cancer. J Clin Oncol 2017;35:3638-46.

7. Hortobagyi GN, Stemmer SM, Burris HA, et al. Ribociclib as First-Line Therapy for HR-Positive, Advanced Breast Cancer. N Engl J Med 2016;375:1738-48.

8. Jiang $\mathrm{Z}, \mathrm{Li} \mathrm{W}, \mathrm{Hu} \mathrm{X}$, et al. Tucidinostat plus exemestane for postmenopausal patients with advanced, hormone 
receptor-positive breast cancer (ACE): a randomised, double-blind, placebo-controlled, phase 3 trial. Lancet Oncol 2019;20:806-15.

9. André F, Ciruelos E, Rubovszky G, et al. Alpelisib for PIK3CA-Mutated, Hormone Receptor-Positive Advanced Breast Cancer. N Engl J Med 2019;380:1929-40.

10. Malumbres M, Barbacid M. Cell cycle, CDKs and cancer: a changing paradigm. Nat Rev Cancer 2009;9:153-66.

11. Otto T, Sicinski P. Cell cycle proteins as promising targets in cancer therapy. Nat Rev Cancer 2017;17:93-115.

12. Altucci L, Addeo R, Cicatiello L, et al. 17beta-Estradiol induces cyclin D1 gene transcription, p36D1-p34cdk4 complex activation and $\mathrm{p} 105 \mathrm{Rb}$ phosphorylation during mitogenic stimulation of $\mathrm{G}(1)$-arrested human breast cancer cells. Oncogene 1996;12:2315-24.

13. Meyerson M, Harlow E. Identification of G1 kinase activity for cdk6, a novel cyclin D partner. Mol Cell Biol 1994;14:2077-86.

14. Fornier MN, Rathkopf D, Shah M, et al. Phase I dosefinding study of weekly docetaxel followed by flavopiridol for patients with advanced solid tumors. Clin Cancer Res 2007;13:5841-6.

15. Gallorini M, Cataldi A, di Giacomo V. Cyclin-dependent kinase modulators and cancer therapy. BioDrugs 2012;26:377-91.

16. Nemunaitis JJ, Small KA, Kirschmeier P, et al. A firstin-human, phase 1, dose-escalation study of dinaciclib, a novel cyclin-dependent kinase inhibitor, administered weekly in subjects with advanced malignancies. J Transl Med 2013;11:259.

17. Gelbert LM, Cai S, Lin X, et al. Preclinical characterization of the CDK4/6 inhibitor LY2835219: in-vivo cell cycle-dependent/independent anti-tumor activities alone/in combination with gemcitabine. Invest New Drugs 2014;32:825-37.

18. Finn RS, Dering J, Conklin D, et al. PD 0332991, a selective cyclin D kinase 4/6 inhibitor, preferentially inhibits proliferation of luminal estrogen receptor-positive human breast cancer cell lines in vitro. Breast Cancer Res 2009;11:R77.

19. Tripathy D, Bardia A, Sellers WR. Ribociclib (LEE011): Mechanism of Action and Clinical Impact of This Selective Cyclin-Dependent Kinase 4/6 Inhibitor in Various Solid Tumors. Clin Cancer Res 2017;23:3251-62.

20. Chong QY, Kok ZH, Bui NL, et al. A unique CDK4/6 inhibitor: Current and future therapeutic strategies of abemaciclib. Pharmacol Res 2020;156:104686.

21. Torres-Guzmán R, Calsina B, Hermoso A, et al. Preclinical characterization of abemaciclib in hormone receptor positive breast cancer. Oncotarget 2017;8:69493-507.

22. Asghar U, Witkiewicz AK, Turner NC, et al. The history and future of targeting cyclin-dependent kinases in cancer therapy. Nat Rev Drug Discov 2015;14:130-46.

23. Heckler M, Ali LR, Clancy-Thompson E, et al. Inhibition of CDK4/6 Promotes CD8 T-cell Memory Formation. Cancer Discov 2021;11:2564-81.

24. Goel S, DeCristo MJ, Watt AC, et al. CDK4/6 inhibition triggers anti-tumour immunity. Nature 2017;548:471-5.

25. Patnaik A, Rosen LS, Tolaney SM, et al. Efficacy and Safety of Abemaciclib, an Inhibitor of CDK4 and CDK6, for Patients with Breast Cancer, Non-Small Cell Lung Cancer, and Other Solid Tumors. Cancer Discov 2016;6:740-53.

26. Dickler MN, Tolaney SM, Rugo HS, et al. MONARCH 1, A Phase II Study of Abemaciclib, a CDK4 and CDK6 Inhibitor, as a Single Agent, in Patients with Refractory HR+/HER2- Metastatic Breast Cancer. Clin Cancer Res 2017;23:5218-24.

27. Sledge GW Jr, Toi M, Neven P, et al. MONARCH 2: Abemaciclib in Combination With Fulvestrant in Women With HR+/HER2- Advanced Breast Cancer Who Had Progressed While Receiving Endocrine Therapy. J Clin Oncol 2017;35:2875-84.

28. Johnston S, Martin M, Di Leo A, et al. MONARCH 3 final PFS: a randomized study of abemaciclib as initial therapy for advanced breast cancer. NPJ Breast Cancer 2019;5:5.

29. Zhang J, Yang N, Ji D, et al. A Randomized Phase I Study of Abemaciclib in Chinese Patients with Advanced and/or Metastatic Cancers. Target Oncol 2021;16:177-87.

30. Breast cancer screening guideline for Chinese women. Cancer Biol Med 2019;16:822-4.

31. Zhang QY, Sun T, Yin YM, et al. MONARCH plus: abemaciclib plus endocrine therapy in women with HR+/HER2 - advanced breast cancer: the multinational randomized phase III study. Ther Adv Med Oncol 2020;12:1758835920963925.

32. Chinese Society of Clinical Oncology guidelines for diagnosis and treatment of breast cancer (2021). Beijing: People's Medical Publishing House, 2021.

33. Goel S, Wang Q, Watt AC, et al. Overcoming Therapeutic Resistance in HER2-Positive Breast Cancers with CDK4/6 Inhibitors. Cancer Cell 2016;29:255-69.

34. Tolaney SM, Wardley AM, Zambelli S, et al. Abemaciclib plus trastuzumab with or without fulvestrant versus 
trastuzumab plus standard-of-care chemotherapy in women with hormone receptor-positive, HER2-positive advanced breast cancer (monarcHER): a randomised, open-label, phase 2 trial. Lancet Oncol 2020;21:763-75.

35. Johnston SRD, Harbeck N, Hegg R, et al. Abemaciclib Combined With Endocrine Therapy for the Adjuvant Treatment of HR+, HER2-, Node-Positive, HighRisk, Early Breast Cancer (monarchE). J Clin Oncol 2020;38:3987-98.

36. FDA. FDA approves abemaciclib with endocrine therapy for early breast cancer. 2021. Available online: https:// www.fda.gov/drugs/resources-information-approveddrugs/fda-approves-abemaciclib-endocrine-therapy-earlybreast-cancer (Accessed 08/11/2021).

37. A Study of Abemaciclib (LY2835219) Plus Hormone Therapy in Participants With Early Breast Cancer (eMonarcHER). 2021. Available online: https:// clinicaltrials.gov/ct2/show/NCT04752332? term=eMonarc HER\&draw=2\&rank=1

38. European Medicines Agency. Abemaciclib (Verzenios) assessment report 26 July 2018, EMA/551438/2018. 2019. Available online: https://www.ema.europa.eu/en/ medicines/human/EPAR/verzenios\#product-informationsection

doi: 10.21037/tbcr-21-36

Cite this article as: Yang L, Chen Y, Wang N, Han W. A narrative review of the clinical development of CDK4/6 inhibitor abemaciclib in breast cancer. Transl Breast Cancer Res 2022;3:4.
39. FDA. Labels for NDA 208716-Label (PDF) submission date 03/30/2020. Available online: https://www.accessdata. fda.gov/scripts/cder/daf/index.cfm? event=overview. process\&ApplNo=208716

40. Gervaso L, Montero AJ, Jia X, et al. Venous thromboembolism in breast cancer patients receiving cyclin-dependent kinase inhibitors. J Thromb Haemost 2020;18:162-8.

41. Wright MD, Abraham MJ. Preclinical discovery and development of abemaciclib used to treat breast cancer. Expert Opin Drug Discov 2021;16:485-96.

42. Roberto M, Astone A, Botticelli A, et al. CDK4/6 Inhibitor Treatments in Patients with Hormone Receptor Positive, Her2 Negative Advanced Breast Cancer: Potential Molecular Mechanisms, Clinical Implications and Future Perspectives. Cancers (Basel) 2021;13:332.

43. Wang B, Li R, Wu S, et al. Breast Cancer Resistance to Cyclin-Dependent Kinases 4/6 Inhibitors: Intricacy of the Molecular Mechanisms. Front Oncol 2021;11:651541.

44. Al-Qasem AJ, Alves CL, Ditzel HJ. Resistance Mechanisms to Combined CDK4/6 Inhibitors and Endocrine Therapy in ER+/HER2- Advanced Breast Cancer: Biomarkers and Potential Novel Treatment Strategies. Cancers (Basel) 2021;13:5397. 